\title{
Response of epilithic diatom communities to environmental gradients in subtropical temperate Brazilian rivers
}

\author{
Eduardo A. Lobo ${ }^{1, *}$, Carlos E. Wetzel ${ }^{2,3}$, Luc Ector $^{3}$, Kazuhiro Katoh ${ }^{4}$, Saúl Blanco ${ }^{3,5}$ and \\ Shigeki Mayama ${ }^{6}$
}

${ }^{1}$ Laboratory of Limnology, University of Santa Cruz do Sul, Av. Independência 2293, CEP 96815-900, Santa Cruz do Sul, RS, Brazil.

${ }^{2}$ Laboratory of Limnology, University of Santa Cruz do Sul, RS, Brazil. Present address: Instituto de Botânica, Seção de Ecologia, Av. Miguel Stéfano 3687, CEP 04301-012, São Paulo, SP, Brazil.

${ }^{3}$ Department of Environment and Agro-Biotechnologies (EVA), Public Research Centre-Gabriel Lippmann, Rue du Brill 41, L-4422 Belvaux, Grand-duchy of Luxembourg.

${ }^{4}$ Graduate School of Agriculture and Life Science, The University of Tokyo. Yayoi 1-1-1, Bunkyo-Ku, Tokyo 113-8657, Japan.

${ }^{5}$ Department of Biodiversity and Environmental Management, University of León, E-24071 León, Spain.

${ }^{6}$ Department of Biology, Tokyo Gakugei University, Nukuikita-machi, Koganei-shi, 4-1-1, Tokyo 184, Japan.

* Corresponding author: lobo@unisc.br

Received: 14/7/09

Accepted: $28 / 6 / 10$

\begin{abstract}
Response of epilithic diatom communities to environmental gradients in subtropical temperate Brazilian rivers

This work aims to analyse the response of epilithic diatom communities to environmental gradients in subtropical temperate southern Brazilian rivers to contribute to the development of a widely applicable methodology for water-quality monitoring. Samples for physical, chemical and biological determination were collected monthly, from December 2001 to November 2002 and from March 2003 to February 2004, at 9 stations along the rivers Pardo and Pardinho in the hydrographical basin of Rio Pardo, State of Rio Grande do Sul (RS), Brazil. Physical and chemical variables (water temperature, pH, dissolved oxygen, biochemical oxygen demand, chemical oxygen demand, nitrates, nitrites, phosphates and total dissolved solids) and biological variables (epilithic diatom communities) were used as parameters for water-quality assessment. The data matrix was examined by means of a multivariate ordination using Detrended Correspondence Analysis (DCA). In total, 270 taxa were identified to specific or infra-specific levels. The results showed that of all the physical and chemical variables used, the main gradient along the DCA first axis was eutrophication, which was indicated by its significant correlation with phosphates $(p<0.001)$. Hence, the species' scores on the DCA axis were used as an operational criterion for indicating their tolerance to eutrophication. From this analysis, the 10 taxa that were the most tolerant to eutrophication were: Cyclotella meneghiniana, Fallacia monoculata, Nitzschia acicularis, N. clausii, N. nana, N. palea, Nitzschia sp., Pinnularia sp., Sellaphora pupula sensu lato and Ulnaria acus. The present study showed that epilithic diatom assemblages reflect anthropogenic changes in hydrographic basins in subtropical temperate streams, especially pollution by organic enrichment and eutrophication.
\end{abstract}

Key words: Diatoms, eutrophication, biomonitoring, southern Brazilian rivers.

\section{RESUMEN}

Respuesta de la comunidad de diatomeas epilíticas a gradientes ambientales en ríos brasileños templados subtropicales

Este trabajo pretende analizar la respuesta de la comunidad de diatomeas epilíticas a gradientes ambientales en ríos brasileños templados subtropicales, con el objetivo de contribuir al desarrollo de una metodología ampliamente aplicable para la monitorización de la calidad del agua. Diversas muestras para determinaciones físicas, químicas y biológicas fueron recogidas mensualmente, de diciembre de 2001 a noviembre de 2002, y de marzo de 2003 a febrero de 2004, en nueve estaciones de muestreo distribuidas a lo largo de los ríos Pardo y Pardinho, localizados en la Cuenca Hidrográfica del Río Pardo, Estado del Río Grande del Sur, Brasil. Variables físicas y químicas (temperatura del agua, pH, oxígeno disuelto, demanda 
bioquímica de oxígeno, demanda química de oxígeno, nitratos, nitritos, fosfato y sólidos totales disueltos) y biológicas (comunidad de diatomeas epilíticas) fueron usadas como parámetros para evaluar la calidad del agua. El conjunto de datos fue analizado por medio de una ordenación multivariante usando el Análisis de Correspondencias sin Tendencias (DCA). En total, 270 taxones fueron identificados a nivel específico o infra-específico. Los resultados indicaron que, entre todas las variables físicas y químicas usadas, el principal gradiente establecido a lo largo del primer eje DCA fue la eutrofización, señalada por su correlación significativa con la concentración de fosfatos ( $\mathrm{p}<0.001)$. De esta forma, los valores alcanzados por las especies en el primer eje DCA fueron usados como un criterio operativo para indicar su tolerancia a la eutrofización. Los 10 taxones más tolerantes a la eutrofización fueron: Cyclotella meneghiniana, Fallacia monoculata, Nitzschia acicularis, N. clausii, N. nana, N. palea, Nitzschia sp., Pinnularia sp., Sellaphora pupula sensu lato y Ulnaria acus. El presente estudio muestra que la comunidad de diatomeas epilíticas refleja cambios antropogénicos en ríos templados subtropicales de cuencas hidrográficas, especialmente la contaminación por enriquecimiento orgánico y eutrofización.

Palabras clave: Diatomeas, eutrofización, biomonitorizacion, ríos brasileños del sur.

\section{INTRODUCTION}

High population densities and a multiplicity of industrial and agricultural activities expose most of southern Brazilian hydrographical basins to heavy and increasing environmental impacts, especially pollution by organic enrichment, fertilizers, heavy metals and agrochemical residues. This situation has led to the development of chemical and biological methods to evaluate contamination levels in running waters.

The first approach involves the assessment of physical and chemical variables of lotic systems; however, in most cases, this method allows only instantaneous measurements and therefore restricts knowledge of water conditions to the period when the measurements were taken. The chemistry at any given time is a photograph of the water quality at the time of sampling and ignores temporal variations in waterquality variables, which are usually high in lotic environments (Rocha, 1992).

By integrating the effects of anthropogenic and natural influences, information derived from the use of bioindicators provides a more refined assessment of water quality than physical and chemical measures used alone (Lobo \& Callegaro, 2000). Researchers around the world (e.g., Armitage, 1995) argue that the traditional methods of classification of waterways based on phys- ical, chemical and bacteriological measurements are not sufficient to meet their multiple uses and are particularly deficient for the assessment of aesthetic quality, recreational use and ecological environment, and therefore an integrated quality analysis is needed that considers not only the traditional methods of evaluation, but the biological system. To this end, different biological communities have been used for assessing and monitoring freshwater quality. Among them, benthic diatoms are recognized worldwide as indicators of organic pollution and eutrophication (Kelly \& Whitton, 1995; Gomez \& Licursi, 2001; Lobo et al., 2004a; Ector \& Rimet, 2005). In Brazil, however, little attention has been paid to the use of this group of algae as bioindicators, and only few related studies have been carried out, mainly in the southern region (Lobo et al., 1996, 2002, 2004a,b,c; Oliveira et al., 2001; Wetzel et al., 2002; Hermany et al., 2006; Salomoni et al., 2006; Schneck et al., 2007).

In this context, the main purpose of this study was to analyse the response of epilithic diatom communities to environmental gradients in southern Brazilian rivers, which should contribute to the development of a widely applicable methodology for water-quality monitoring in subtropical temperate regions and provide a scientific basis for the implementation of diatom-based diagnoses of the ecological status of these ecosystems. 


\section{MATERIAL AND METHODS}

\section{Study Area}

Monthly sampling surveys, from December 2001 to November 2002 (stations S1-S6) and March 2003 to February 2004 (stations S2, S6-S9), were made along the Pardo and Pardinho Rivers (Rio Pardo hydrographical Basin), located approximately in the centre of the State of Rio Grande do Sul (RS), Brazil (30 $\left.15^{\prime} 46^{\prime \prime} \mathrm{S}, 5^{\circ} 27^{\prime} 28^{\prime \prime} \mathrm{W}\right)$ (Fig. 1 and Table 1). With a length of $115 \mathrm{~km}$ and an average width of $35 \mathrm{~km}$, the total area of the basin is $3749 \mathrm{~km}^{2}$. Main subbasins correspond to the Pardo and Pardinho Rivers, with areas of
$2660 \mathrm{~km}^{2}(71 \%)$ and $1089 \mathrm{~km}^{2}(29 \%)$, respectively. Santa Cruz do Sul, the main industrial municipal district of the region with a population of 107,000 inhabitants (50\% of the total population lives within the basin area), is situated on the lower reach of the basin. According to Maluf (2000), the annual mean air temperatures in the study area $\left(14\right.$ to $\left.20^{\circ} \mathrm{C}\right)$ characterise it as a subtropical temperate region.

\section{Sampling/Analytical Procedures}

Physical, chemical, and biological variables were used as parameters for assessing water quality. The following physical and chemical variables

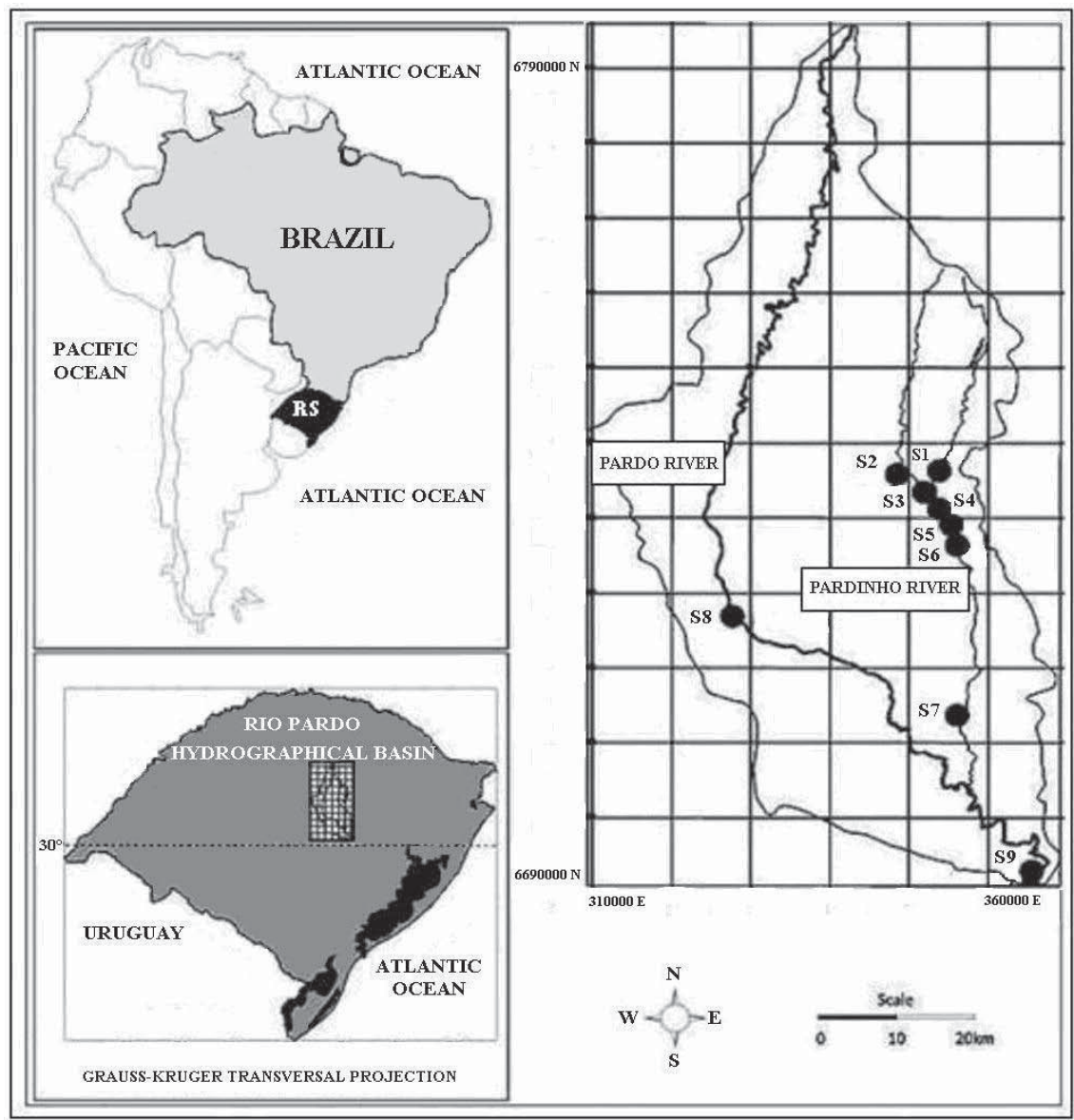

Figure 1. Rio Pardo hydrographical basin in the State of Rio Grande do Sul (RS), Brazil, with the location of sampling sites in the Rio Pardinho (S1-S7) and Rio Pardo subbasins (S8-S9). Cuenca Hidrográfica del Río Pardo en el Estado del Río Grande del Sur (RS), Brasil, mostrando la localización de los sitios de muestreo en la subcuenca del Río Pardinho (S1-S7) y en la subcuenca del Río Pardo (S8-S9). 
Table 1. Location of sampling sites in the Rio Pardo Hydrographical Basin, RS, Brazil. Localización de los sitios de muestreo en la Cuenca Hidrográfica del Río Pardo, RS, Brasil.

\begin{tabular}{llll}
\hline Code & River Name & \multicolumn{2}{c}{ Geographic Coordinates } \\
\hline S 1 & Rio Pequeno & $52^{\circ} 31^{\prime} 04.20^{\prime \prime} \mathrm{W}$ & $29^{\circ} 29^{\prime} 54.76^{\prime \prime} \mathrm{S}$ \\
S 2 & Rio Pardinho & $52^{\circ} 33^{\prime} 15.44^{\prime \prime} \mathrm{W}$ & $29^{\circ} 30^{\prime} 05.39^{\prime \prime} \mathrm{S}$ \\
S 3 & Rio Pardinho & $52^{\circ} 31^{\prime} 00.69^{\prime \prime} \mathrm{W}$ & $29^{\circ} 31^{\prime} 30.86^{\prime \prime} \mathrm{S}$ \\
S 4 & Rio Pardinho & $52^{\circ} 31^{\prime} 07.74^{\prime \prime} \mathrm{W}$ & $29^{\circ} 31^{\prime} 59.98^{\prime \prime} \mathrm{S}$ \\
S 5 & Rio Pardinho & $52^{\circ} 30^{\prime} 15.57^{\prime \prime} \mathrm{W}$ & $29^{\circ} 32^{\prime} 44.36^{\prime \prime} \mathrm{S}$ \\
S 6 & Rio Pardinho & $52^{\circ} 29^{\prime} 07.80^{\prime \prime} \mathrm{W}$ & $29^{\circ} 33^{\prime} 11.60^{\prime \prime} \mathrm{S}$ \\
S 7 & Rio Pardinho & $52^{\circ} 29^{\prime} 10.23^{\prime \prime} \mathrm{W}$ & $29^{\circ} 48^{\prime} 00.04^{\prime \prime} \mathrm{S}$ \\
S 8 & Rio Pardo & $52^{\circ} 46^{\prime} 12.31^{\prime \prime} \mathrm{W}$ & $29^{\circ} 40^{\prime} 22.58^{\prime \prime} \mathrm{S}$ \\
S 9 & Rio Pardo & $52^{\circ} 22^{\prime} 56.90^{\prime \prime} \mathrm{W}$ & $29^{\circ} 58^{\prime} 35.31^{\prime \prime} \mathrm{S}$ \\
\hline
\end{tabular}

were used: water temperature $\left(\mathrm{T} ;{ }^{\circ} \mathrm{C}\right), \mathrm{pH}$, dissolved oxygen (DO; $\left.\mathrm{mg} \mathrm{L}^{-1}\right)$, biochemical oxygen demand after 5 days $\left(\mathrm{BOD}_{5} ; \mathrm{mg} \mathrm{L}^{-1}\right)$, chemical oxygen demand (COD; $\left.\mathrm{mg} \mathrm{L}^{-1}\right)$, nitrates $\left(\mathrm{NO}_{3}^{-} ; \mathrm{mg} \mathrm{L}^{-1}\right)$, nitrites $\left(\mathrm{NO}_{2}^{-} ; \mathrm{mg} \mathrm{L}^{-1}\right)$, phosphates $\left(\mathrm{PO}_{4}^{3-} ; \mathrm{mg} \mathrm{L}^{-1}\right)$ and total dissolved solids (TDS; $\mathrm{mg} \mathrm{L}^{-1}$ ). Sampling protocols for physical and chemical analyses followed those described in American Public Health Association (1999).

Epilithic algal samples for diatom identification and quantification were taken monthly, along with measurements of physical and chemical variables. For qualitative and semi-quantitative analyses, samples were scrubbed off the upper surface of submerged stones that were $10-20 \mathrm{~cm}$ in diameter using a toothbrush and fixed with formalin following the method of Kobayasi \& Mayama (1982). Diatom samples were cleaned with sulphuric and hydrochloric acids and mounted on microscopic slides with Pleurax ${ }^{R}$. All individuals found in random transects under light microscopy (LM) across each permanent slide were identified and counted, up to a minimum of 600 valves, using an Olympus BX-40 microscope. For species identification, the following taxonomical sources were used: Krammer \& Lange-Bertalot (1986, 1988, 1991a, b), Metzeltin \& Lange-Bertalot (1998, 2002, 2007), Krammer (2000), Rumrich et al. (2000), Lobo et al. (2002, 2004a) and Metzeltin et al. (2005). Quantitatively important species, or abundant species, were indicated following the criterion of Lobo \& Leighton (1986). Voucher samples were stored in the DIAT-UNISC Herbarium at the University of Santa Cruz do Sul, RS, Brazil.
Data matrices (species counts and environmental variables) were examined by means of a multivariate ordination using Detrended Correspondence Analysis (DCA, Hill \& Gauch, 1980), because it does not produce the arc or horseshoe effect, a spurious second axis which is a curvilinear function of the first axis of Correspondence Analysis (CA) or Principal Component Analysis (PCA). The score of each species on the DCA axes was used as an operational criterion for indicating its tolerance of eutrophication. Therefore, species with scores above 250 were classified as "eutrophication tolerant taxa". Species appearing in 12 samples or less were omitted from the evaluation due to the low reliability of their scores. The biological matrix was also log-transformed $[\log (x+1)]$ and analysed by means of DCA using river reaches as categorical predictors for plotting sampling sites located in the middle (S1-S6) and lowland reaches (S7-S9). Statistical analyses were performed using PC-ORD software version 4.2 (McCune \& Mefford, 1999).

\section{RESULTS AND DISCUSSION}

A total of 270 taxa belonging to 53 genera were identified to generic, specific or infra-specific levels, 66 of which were abundant (Table 2). The genera Nitzschia (12.2\%), Navicula (11.5\%) and Gomphonema (7.4\%) had the highest number of species, followed by Eunotia $(7.0 \%)$ and Pinnularia (5.2\%).

Table 3 shows the annual mean and standard deviation for the environmental variables at the 
Table 2. Maximum Relative Abundance (MRA, \%) and Frequency of Occurrence (FO, \%) of the 66 abundant diatom taxa found in the Rio Pardo Hydrographical Basin, RS, Brazil. Saprobic value (s) (Lobo et al., 2002), and indicative value (vi) (Lobo et al., 2004a) are also given. Abundancia relativa máxima-MRA (\%) y frecuencia de ocurrencia-FO (\%) de las 66 especies de diatomeas abundantes encontradas en la Cuenca Hidrográfica del Río Pardo, RS, Brasil. Se ofrecen también los valores sapróbicos (s) (Lobo et al., 2002) y los valores indicativos (Lobo et al., 2004a).

\begin{tabular}{|c|c|c|c|c|}
\hline Taxon & $s$ & $v i$ & MRA & FO \\
\hline Achnanthes inflata (Kützing) Grunow & 2.5 & 1 & 3.3 & 17.1 \\
\hline Achnanthidium exiguum var. constrictum (Grunow) N.A. Andresen, Stoermer et Kreis & 2.5 & 5 & 34.5 & 96.1 \\
\hline Achnanthidium minutissimum (Kützing) Czarnecki & 2.5 & 3 & 52.8 & 91.5 \\
\hline Adlafia drouetiana (R.M. Patrick) Metzeltin et Lange-Bertalot & 4.0 & 3 & 4.8 & 43.4 \\
\hline Amphipleura lindheimeri Grunow & 4.0 & 3 & 3.3 & 18.6 \\
\hline Amphora montana Krasske & 2.5 & 1 & 10.1 & 32.6 \\
\hline Cocconeis euglypta (Ehrenberg) Grunow & 2.5 & 3 & 2.1 & 24.0 \\
\hline Cocconeis lineata (Ehrenberg) Van Heurck & 2.5 & 2 & 8.2 & 48.1 \\
\hline Cyclotella meneghiniana Kützing & 2.5 & 3 & 4.8 & 27.9 \\
\hline Diadesmis confervacea Kützing & 2.5 & 1 & 3.4 & 50.4 \\
\hline Diadesmis contenta (Grunow ex Van Heurck) D.G. Mann & 2.5 & 3 & 38.7 & 65.1 \\
\hline Encyonema silesiacum (Bleisch) D.G. Mann & 2.5 & 2 & 16.7 & 70.5 \\
\hline Encyonema sprechmanii Metzeltin et al. & 1.0 & 1 & 4.3 & 23.3 \\
\hline Eolimna minima (Grunow) Lange-Bertalot & 1.0 & 4 & 3.1 & 34.1 \\
\hline Eolimna subminuscula (Manguin) Gerd Moser, Lange-Bertalot et Metzeltin & 1.0 & 1 & 11.9 & 61.2 \\
\hline Fallacia monoculata (Hustedt) D.G. Mann & 4.0 & 5 & 22.2 & 17.1 \\
\hline Fragilaria rumpens (Kützing) G.W.F. Carlson & 2.5 & 4 & 20.4 & 20.2 \\
\hline Frustulia crassinervia (Brébisson) Lange-Bertalot et Krammer & 1.0 & 1 & 3.7 & 15.5 \\
\hline Geissleria aikenensis (R.M. Patrick) Torgan et Oliveira & 2.5 & 1 & 55.5 & 100.0 \\
\hline Gomphonema affine Kützing & 1.0 & 1 & 0.9 & 16.3 \\
\hline Gomphonema angustatum (Kützing) Rabenhorst & 2.5 & 4 & 11.4 & 76.7 \\
\hline Gomphonema aff. clevei Fricke & 4.0 & 3 & 5.5 & 28.7 \\
\hline Gomphonema gracile Ehrenberg & 2.5 & 1 & 2.2 & 24.8 \\
\hline Gomphonema mexicanum Grunow & 1.0 & 1 & 2.4 & 38.0 \\
\hline Gomphonema parvulum (Kützing) Kützing & 2.5 & 4 & 25.1 & 96.1 \\
\hline Gomphonema pseudoaugur Lange-Bertalot & 4.0 & 1 & 9.0 & 23.3 \\
\hline Gomphonema pumilum (Grunow) E. Reichardt et Lange-Bertalot & 1.0 & 1 & 22.7 & 55.8 \\
\hline Gomphonema turris var. brasiliensis (Fricke in Schmidt et al.) Frenguelli & 1.0 & 1 & 3.8 & 20.9 \\
\hline Gyrosigma acuminatum (Kützing) Rabenhorst & 2.5 & 1 & 7.6 & 19.4 \\
\hline Gyrosigma scalproides (Rabenhorst) Cleve & 4.0 & 1 & 5.2 & 49.6 \\
\hline Luticola goeppertiana (Bleisch) D.G. Mann & 4.0 & 1 & 7.4 & 45.0 \\
\hline Luticola mutica (Kützing) D.G. Mann & 1.0 & 1 & 3.9 & 33.3 \\
\hline Mayamaea atomus (Kützing) Lange-Bertalot & 2.5 & 5 & 41.4 & 76.0 \\
\hline Melosira varians C. Agardh & 4.0 & 3 & 6.3 & 21.7 \\
\hline Navicula cruxmeridionalis Metzeltin, Lange-Bertalot et García-Rodríguez & 1.0 & 1 & 8.6 & 7.0 \\
\hline Navicula cryptocephala Kützing & 2.5 & 1 & 5.7 & 88.4 \\
\hline Navicula cryptotenella Lange-Bertalot & 4.0 & 3 & 17.4 & 92.2 \\
\hline Navicula gregaria Donkin & 2.5 & 3 & 19.8 & 74.4 \\
\hline Navicula notha J.H. Wallace & 1.0 & 1 & 1.8 & 13.2 \\
\hline Navicula pseudoarvensis Hustedt & 1.0 & 1 & 2.7 & 18.6 \\
\hline Navicula rostellata Kützing & 2.5 & 4 & 14.6 & 89.1 \\
\hline Navicula symmetrica R.M. Patrick & 4.0 & 3 & 9.4 & 81.4 \\
\hline
\end{tabular}


Table 2. (cont.)

\begin{tabular}{|c|c|c|c|c|}
\hline Taxon & $s$ & $v i$ & MRA & FO \\
\hline Naviculadicta sp. & 1.0 & 1 & 1.9 & 11.6 \\
\hline Nitzschia acicularis (Kützing) W. Smith & 1.0 & 1 & 3.7 & 22.5 \\
\hline Nitzschia amphibia Grunow & 4.0 & 2 & 28.8 & 93.8 \\
\hline Nitzschia brevissima Grunow & 1.0 & 1 & 1.6 & 3.9 \\
\hline Nitzschia clausii Hantzsch & 1.0 & 1 & 6.3 & 30.2 \\
\hline Nitzschia frustulum (Kützing) Grunow & 1.0 & 1 & 7.4 & 20.2 \\
\hline Nitzschia intermedia Hantzsch ex Cleve and Grunow & 1.0 & 1 & 5.2 & 7.0 \\
\hline Nitzschia linearis (C. Agardh) W. Smith & 4.0 & 3 & 3.4 & 41.1 \\
\hline Nitzschia nana Grunow & 4.0 & 1 & 9.6 & 12.4 \\
\hline Nitzschia palea (Kützing) W. Smith & 4.0 & 3 & 52.7 & 98.4 \\
\hline Nitzschia pumila Hustedt & 1.0 & 1 & 2.5 & 3.9 \\
\hline Nitzschia sp. & 1.0 & 1 & 3.6 & 10.9 \\
\hline Nupela praecipua (E. Reichardt) E. Reichardt & 1.0 & 1 & 3.6 & 17.1 \\
\hline Pinnularia latarea Krammer & 1.0 & 1 & 1.3 & 28.7 \\
\hline Pinnularia sp. & 1.0 & 1 & 3.2 & 12.4 \\
\hline Planothidium lanceolatum (Brébisson ex Kützing) Lange-Bertalot & 2.5 & 1 & 44.7 & 87.6 \\
\hline Planothidium rostratum (Lange-Bertalot) Lange-Bertalot & 4.0 & 2 & 5.2 & 74.4 \\
\hline Platessa hustedtii (Krasske) Lange-Bertalot & 1.0 & 1 & 7.6 & 52.7 \\
\hline Sellaphora pupula (Kützing) Mereschkowsky sensu lato & 1.0 & 1 & 5.9 & 64.3 \\
\hline Sellaphora rhombicarea Metzeltin, Lange-Bertalot et García-Rodríguez & 4.0 & 1 & 3.1 & 31.8 \\
\hline Sellaphora seminulum (Grunow) D.G. Mann & 2.5 & 5 & 69.1 & 99.2 \\
\hline Surirella angusta Kützing & 2.5 & 1 & 5.2 & 51.9 \\
\hline Ulnaria acus (Kützing) Aboal & 1.0 & 1 & 5.7 & 30.2 \\
\hline Ulnaria ulna (Nitzsch) Compère & 4.0 & 1 & 37.5 & 10.1 \\
\hline
\end{tabular}

nine sampling sites in the Rio Pardo hydrographical basin (RS), which were measured from December 2001 to November 2002 (S1-S6) and from March 2003 to February 2004 (S2, S6-S9).

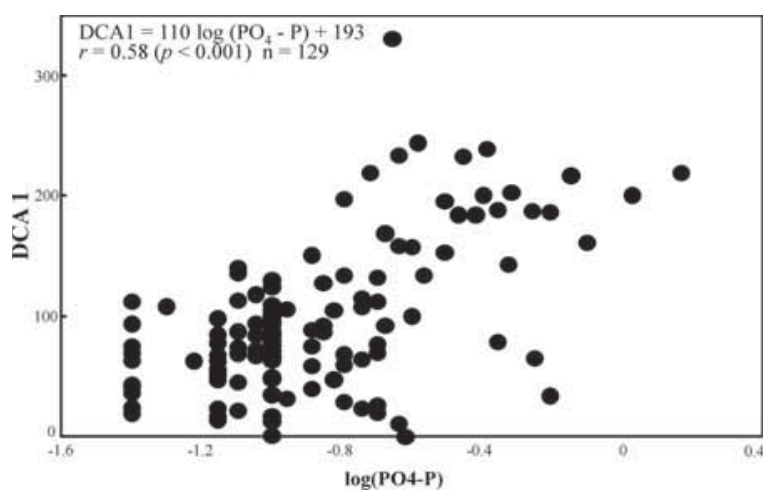

Figure 2. Scatter plot showing the relationship between phosphate concentration and DCA first axis (DCA1) scores. Diagrama de dispersión mostrando la relación entre la concentración de fosfatos y los valores del primer eje del DCA (DCA1).
The species data matrix was examined by means of a multivariate ordination using DCA. The results showed that, of all the physical and

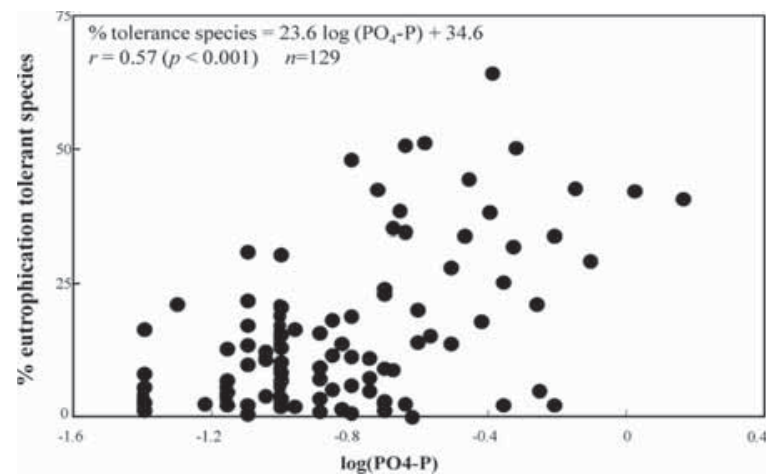

Figure 3. Scatter plot showing the relationship between phosphate concentration and the sum of relative abundances of the main eutrophication-tolerant species. Diagrama de dispersión mostrando la relación entre la concentración de fosfatos y la suma de las abundancias relativas de las principales especies tolerantes a la eutrofización. 
Table 3. Annual mean $(\times)$ and standard deviation (s.d.) $(n=12)$ of environmental variables at sampling sites in the Rio Pardo Hydrographical Basin, RS, Brazil, from December 2001 to November 2002 (S1-S6) and March 2003 to February 2004 (S2, S6S9) (T: water temperature, DO: dissolved oxygen, $\mathrm{BOD}_{5}$ : biochemical oxygen demand, $\mathrm{COD}$ : chemical oxygen demand, $\mathrm{PO}_{4}^{3-}$ : phosphates, $\mathrm{NO}_{3}^{-}$: nitrates, $\mathrm{NO}_{2}^{-}$: nitrites, TDS: total dissolved solids). Promedios anuales $(\times)$y desviaciones típicas (s.d.) ( $\mathrm{n}=12$ ) de las variables ambientales en los sitios de muestreo de la Cuenca Hidrográfica del Río Pardo, RS, Brasil, de diciembre 2001 a noviembre 2002 (S1-S6) y marzo 2003 a febrero 2004 (S2, S6-S9) (T: temperatura del agua, DO: oxígeno disuelto, BOD 5 : demanda bioquímica de oxígeno, $\mathrm{COD}$ : demanda química de oxígeno, $\mathrm{PO}_{4}^{3-}$ : fosfatos, $\mathrm{NO}_{3}^{-}$: nitratos, $\mathrm{NO}_{2}^{-}$: nitritos, TDS: sólidos totales disueltos).

\begin{tabular}{|c|c|c|c|c|c|c|c|c|c|c|}
\hline & tes & $\begin{array}{c}\mathbf{T} \\
\left({ }^{\circ} \mathbf{C}\right)\end{array}$ & $\begin{array}{c}\text { pH } \\
\left(\mathrm{mg} \mathrm{L}^{-1}\right)\end{array}$ & $\begin{array}{c}\text { DO } \\
\left(\mathrm{mg} \mathrm{L}^{-1}\right)\end{array}$ & $\begin{array}{c}\mathrm{BOD}_{5} \\
\left(\mathrm{mg} \mathrm{L}^{-1}\right)\end{array}$ & $\begin{array}{c}\text { COD } \\
\left(\mathrm{mg} \mathrm{L}^{-1}\right)\end{array}$ & $\begin{array}{c}\mathrm{PO}_{4}^{3-} \\
\left(\mathrm{mg} \mathrm{L}^{-1}\right)\end{array}$ & $\begin{array}{c}\mathrm{NO}_{3}^{-} \\
\left(\mathrm{mg} \mathrm{L}^{-1}\right)\end{array}$ & $\begin{array}{c}\mathrm{NO}_{2}^{-} \\
\left(\mathrm{mg} \mathrm{L}^{-1}\right)\end{array}$ & TDS \\
\hline & $(\times)$ & 19.1 & 6.7 & 8.6 & 2.01 & 4.2 & 0.10 & 0.51 & 0.01 & 58.7 \\
\hline SI & (s. d.) & 5.1 & 0.4 & 3.0 & 0.78 & 1.5 & 0.06 & 0.25 & 0.00 & 21.6 \\
\hline & $(\times)$ & 18 & 6.8 & 8.8 & 0.92 & 4.6 & 0.16 & 0.72 & 0.01 & 59.8 \\
\hline 32 & (s. d.) & 4.2 & 0.7 & 1.2 & 1.20 & 3.4 & 0.14 & 0.23 & 0.00 & 18.9 \\
\hline & $(\times)$ & 19.8 & 6.9 & 8.6 & 2.14 & 6.8 & 0.09 & 0.52 & 0.01 & 49.9 \\
\hline 53 & (s. d.) & 4.4 & 0.5 & 0.9 & 0.49 & 7.2 & 0.03 & 0.23 & 0.00 & 13.3 \\
\hline & $(\times)$ & 20.2 & 6.9 & 8.6 & 2.32 & 4.4 & 0.09 & 0.55 & 0.05 & 58.6 \\
\hline S4 & (s. d.) & 5.0 & 0.4 & 0.9 & 0.66 & 1.7 & 0.04 & 0.24 & 0.07 & 10.1 \\
\hline & $(\times)$ & 20.2 & 6.8 & 8.4 & 2.27 & 4.5 & 0.10 & 0.60 & 0.01 & 52.8 \\
\hline 55 & (s. d.) & 5.2 & 0.4 & 1.0 & 0.77 & 2.8 & 0.03 & 0.37 & 0.00 & 12.4 \\
\hline & $(\times)$ & 19.3 & 7.0 & 8.7 & 0.72 & 4.3 & 0.19 & 0.90 & 0.01 & 79.6 \\
\hline S6 & (s. d.) & 4.0 & 0.5 & 1.5 & 0.77 & 3.7 & 0.14 & 0.35 & 0.00 & 25.4 \\
\hline & $(\times)$ & 19.7 & 7.1 & 7.2 & 3.00 & 5.8 & 0.52 & 1.05 & 0.05 & $\overline{118.1}$ \\
\hline 57 & (s. d.) & 5.6 & 0.3 & 1.5 & 2.35 & 3.7 & 0.22 & 0.22 & 0.04 & 23.3 \\
\hline & $(\times)$ & 19.3 & 6.9 & 8.4 & 1.19 & 7.4 & 0.21 & 0.66 & 0.01 & 75.5 \\
\hline S8 & (s. d.) & 5.6 & 0.3 & 1.1 & 1.86 & 7.1 & 0.08 & 0.31 & 0.00 & 18.5 \\
\hline So & $(\times)$ & 20.3 & 6.9 & 7.7 & 1.86 & 6.6 & 0.45 & 0.81 & 0.02 & $\overline{117.4}$ \\
\hline 39 & (s. d.) & 5.7 & 0.4 & 1.0 & 1.58 & 2.6 & 0.41 & 0.25 & 0.01 & 36.9 \\
\hline
\end{tabular}

chemical variables used for pollution assessment, the main gradient expressed by the first axis was eutrophication, which was indicated by the significant correlation $(p<0.001)$ found with phosphates (Fig. 2).

Because the DCA first axis represented eutrophication, the score of each species along this axis was used as an operational criterion for indicating their tolerance of eutrophication. Figure 3 presents the relationship between the concentration of phosphates and the sum of the relative abundances of the 10 main eutrophicationtolerant species, namely: Cyclotella meneghiniana Kützing, Fallacia monoculata (Hustedt) D.G. Mann, Nitzschia acicularis (Kützing) W. Smith, $N$. clausii Hantzsch, $N$. nana Grunow, $N$. palea (Kützing) W. Smith, Nitzschia sp., Pinnularia sp., Sellaphora pupula (Kützing) Mereschkowsky sensu lato and Ulnaria acus (Kützing) Aboal. Light and scanning electron mi- croscopy photographs of these 10 taxa are shown in figures 5-77, along with other frequently observed species such as Sellaphora seminulum (Grunow) D. G. Mann and Luticola goeppertiana (Bleisch) D. G. Mann.

The ordination of sampling sites along the middle and lowland reaches (Fig. 4) showed that sites are arranged following an eutrophication gradient, with the lowland sampling sites grouped together (white circles in Fig. 4) and thus indicating their environmental characteristics. Eutrophication was indicated by the amount of phosphate, and these sites showed an average concentration of $0.39 \pm 0.16 \mathrm{mg} \mathrm{L}^{-1} \mathrm{PO}_{4}^{3-}$ (Table 3), a value two times higher than the limit established by Resolution 357 of the Brazilian Council for the Environment (CONAMA, 2005) to separate water use Class " 4 " from Class " 3 ". This corresponds to the worst quality water uses class, and indicates a waterway that is basi- 


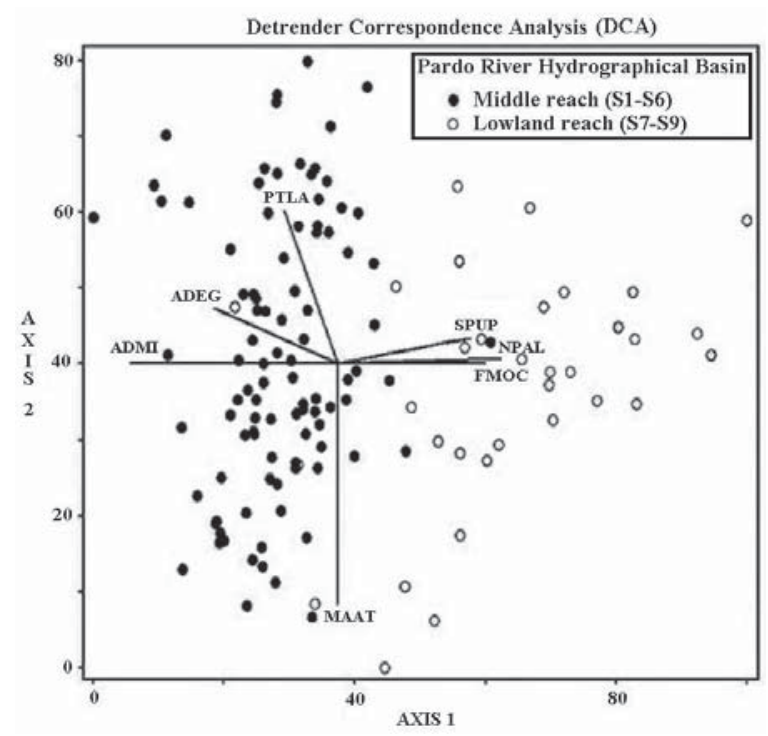

Figure 4. Scatter plot showing the relationship between diatom species and sampling sites located at middle (S1S6) and lowland (S7-S9) reaches. ADEG: Achnanthidium exiguum; ADMI: A. minutissimum; FMOC: Fallacia monoculata; MAAT: Mayamaea atomus; NPAL: Nitzschia palea; PTLA: Planothidium lanceolatum; SPUP: Sellaphora pupula. The cutoff value for joint plot is $\mathrm{r}^{2} \geq 0.400$. Diagrama de dispersión mostrando la relación entre las especies de diatomeas y los sitios de muestreo localizados en los tramos intermedios (S1-S6) e inferiores (S7-S9). ADEG: Achnanthidium exiguum; ADMI: A. minutissimum; FMOC: Fallacia monoculata; MAAT: Mayamaea atomus; NPAL: Nitzschia palea; PTLA: Planothidium lanceolatum; SPUP: Sellaphora pupula. Valor de corte para la graficación $r^{2} \geq 0,400$.

cally utilized for navigation. Yet, the sites can be classified as polysaprobic environment according to Hamm's (1969) water-quality classification (>0.327 mg L $\mathrm{mg}^{-1} \mathrm{PO}_{4}^{3-}$ ). The location of Sellaphora pupula (SPUP), Nitzschia palea (NPAL) and Fallacia monoculata (FMOC) with respect to the sampling sites indicates the trophic preferences of these species.

All eutrophication tolerant diatoms are considered cosmopolitan, according to the classification of Cocquyt (2000). Cyclotella meneghiniana is one of the most thoroughly studied freshwater centric diatoms (e.g., Beszteri et al., 2007), is regarded as the most common species of global diatom diversity and occupies a wide range of habitat types (Håkansson, 2002). According to Denys (1991), C. meneghiniana is a tychoplanktonic species, occurring in brackish and freshwater, eutraphentic, $\alpha$-meso- to polysaprobic envi- ronments (Van Dam et al., 1994). According to Krammer \& Lange-Bertalot (1991a), it is common in ditches and puddles and in eutrophic lakes and rivers. Cells of $C$. meneghiniana can grow in a wide variety of habitats but not in highly competitive situations (Patrick \& Roberts, 1979). In strongly eutrophic and polluted waters, which are presumably free of interspecific competition, C. meneghiniana may develop large populations (Wojtal \& Kwandrans, 2006). According to regional classifications (Lobo et al., 2004a), this species has a high tolerance for organic pollution and an average tolerance for eutrophication. Lobo et al. (2002) classified C. meneghiniana as highly tolerant to organic pollution in the lower reaches of the Rio Pardo hydrographical basin, southern Brazil. Lobo et al. (2004c), while working on urban streams in Porto Alegre County, southern Brazil, confirmed that phosphates were the most important variable in ordination plots (Canonical Correspondence Analysis), which showed an evident eutrophication gradient, with $C$. meneghiniana and Sellaphora pupula being extremely abundant under high nutrient concentrations. According to Salomoni et al. (2006), S. pupula showed high densities at stations closer to the mouth of the Gravataí River, southern Brazil, which corresponded to the most eutrophic zone and was heavily polluted by industrial and domestic wastewater inflows. The preference of $S$. pupula for more eutrophic environments was also seen in southern Brazil by Lobo et al. (2004c) in a study of the urban streams Condor and Capivara in the city of Porto Alegre.

The $S$. pupula species complex is well known for its large morphological variability (Mann, 2001; Mann et al., 2008). Some authors (e.g., Cleve-Euler, 1953) divided it into a number of varieties, but in later studies, these varieties were treated as forms (e.g., Hustedt, 1930-1966) or not even given any taxonomical category (Schoeman \& Archibald, 1976-1980), although these morphodemes seem to be ecologically differentiated (Round, 1972). Furthermore, the application of a broad species concept, as well as the universal use of European floras by diatomists under the assumption that most diatom species are cosmopolitan (Hustedt, 1930-1966; Krammer 


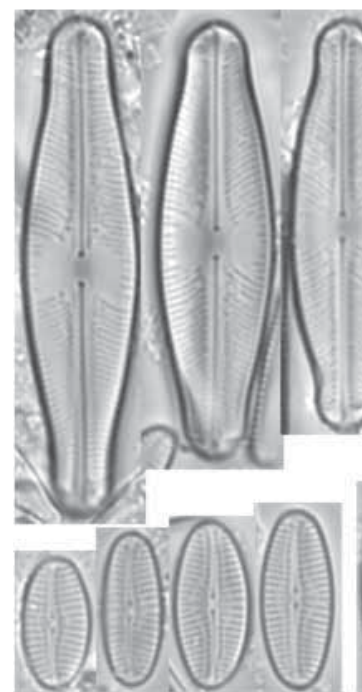

15-18

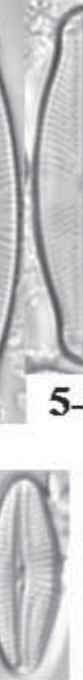

19

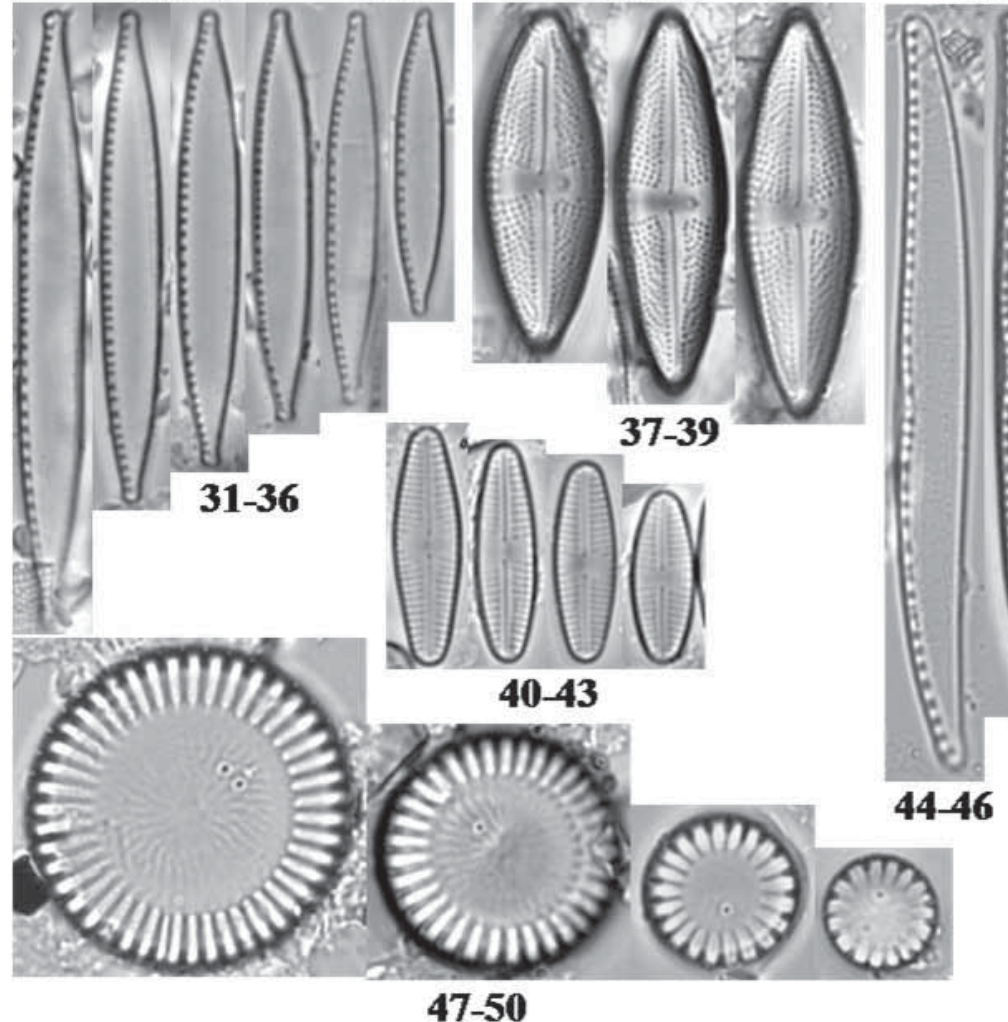

47-50
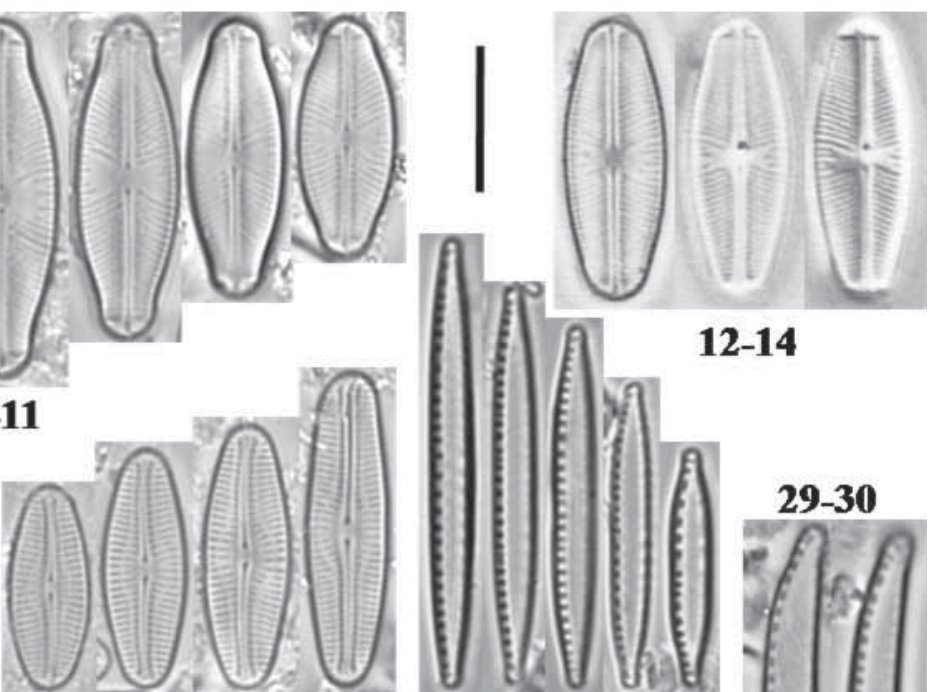

20-23

24-28

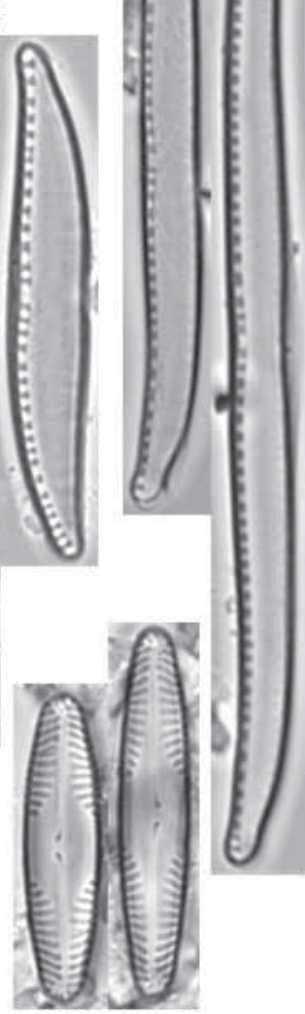

51-52

Figures 5-52. Light microscopy. 5-11: Sellaphora pupula. 12-14: Reproduction of S. pupula lectotype (British Museum, BM 17918) designated by Mann (2001, p. 231, figs. 2-4). 15-23: Fallacia monoculata. 15-18, 20-23: Specimens from the Rio Pardo hydrographical basin. 19: Holotype designated by Hustedt (1945). 24-28: Nitzschia sp. 29-30: N. nana. 31-36: N. palea. 37-39: Luticola goeppertiana. 40-43: Sellaphora seminulum. 44-46: N. clausii. 47-50: Cyclotella meneghiniana. 51-52: Pinnularia sp. Scale bar $=10 \mu \mathrm{m}$. Microscopia óptica. 5-11: Sellaphora pupula. 12-14: Reproducción del lectotipo de S. pupula (British Museum, BM 17918) designado por Mann (2001, p. 231, figs. 2-4). 15-23: Fallacia monoculata. 15-18, 20-23: Especimenes de la Cuenca Hidrográfica del Río Pardo. 19: Holotipo designado por Hustedt (1945). 24-28: Nitzschia sp. 29-30: N. nana. 31-36: N. palea. 37-39: Luticola goeppertiana. 40-43: Sellaphora seminulum. 44-46: N. clausii. 47-50: Cyclotella meneghiniana. 51-52: Pinnularia sp. Barra de escala = $10 \mu \mathrm{m}$. 


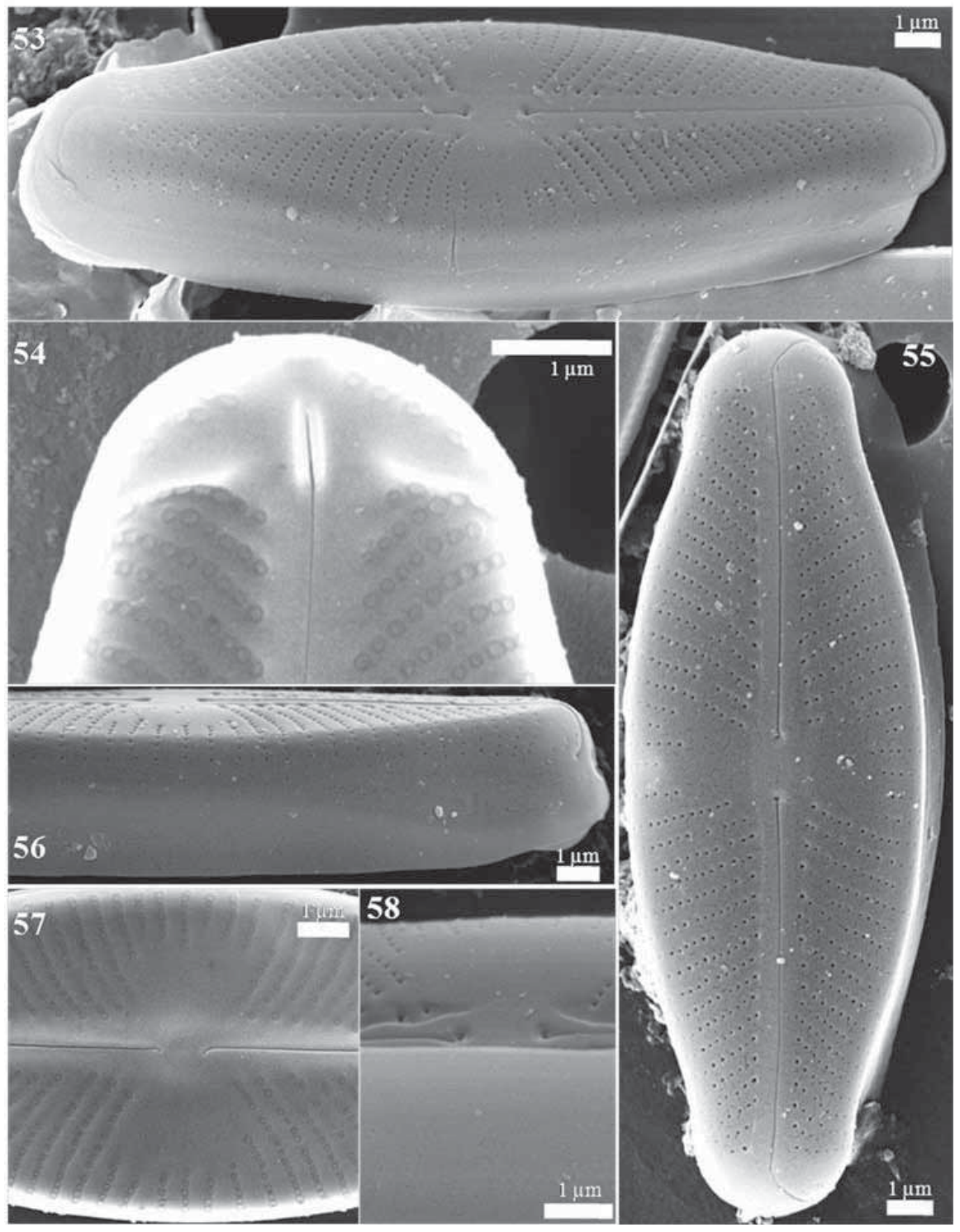

Figures 53-58. Scanning electron microscopy images of Sellaphora pupula. 53: Tilted external view of a valve showing general arrangement of striae and valve plan flat. 54: Internal pole detail showing straight distal raphe end stopping in helictoglossae and internal areolae with closing membranes and highly silicified polar-bars. 55: External view showing general valve patterns, terminal raphe ends curved to the same side, uniseriate striae composed of 5 to 13 areolae, central area bordered by 5 short, transapically butterfly-shaped striae, proximal drop-shaped raphe endings and distal raphe fissures that are strongly curved towards the mantle. 56: Mantle view detail showing the drop-shaped distal raphe end and rounded valve face/mantle transition without interruption of the striae. 57: Internal view of the central area, straight raphe filiform and proximal endings of the raphe gently bent on the same side. 58: External view of the central area showing proximal raphe ends and slightly elevated axial area (sternum). Imágenes de microscopia electrónica de barrido de Sellaphora pupula. 53: Vista externa de la valva inclinada mostrando la ornamentación general de las estrías y un plano horizontal de la valva. 54: Detalle del polo interno, mostrando la extremidad distal del rafe recta, terminando en una helictoglosa, y areolas internas con membranas cerradas, barras polares fuertemente silicificadas. 55: Vista externa mostrando el patrón general de la valva, extremos terminales del rafe curvados al mismo lado; estrías uniseriadas compuestas de 5 a 13 areolas, área central rodeada por 5 estrías cortas, con forma de mariposa transapicalmente; terminación proximal del rafe en forma de gota; fisura distal del rafe fuertemente curvada hacia el manto. 56: Detalle del manto mostrando el extremo distal del rafe en forma de gota; la transición entre la parte valvar y el manto redondeada; sin interrupción de las estrías. 57: Vista interna del área central; rafe filiforme; recto; terminaciones proximales del rafe suavemente curvadas al mismo lado. 58: Vista externa del área central mostrando el extremo proximal del rafe y un área axial ligeramente elevada (esterno). 
\& Lange-Bertalot, 1986, 1988, 1991a,b), has increased taxonomic confusion. Nevertheless according to Mann (2001), since 1990, a narrower species concept has begun to be adopted, with the result that many more species are now being recognised than before, and species delimitation has become correspondingly more difficult. Since the reestablishment of Sellaphora as an independent genus nearly 20 years ago by Mann (1989), the number of species has increased from 8 to 80 (Mann et al., 2008), and a large number of South American species belonging to this genus, mainly from tropical and subtropical waters, has emerged (Metzeltin \& Lange-Bertalot, 1998, 2002, 2007; Metzeltin et al., 2005). Moreover, even in well-studied areas such as central and northern Europe, there are still a large number of species yet to be described. Clearly, a more detailed study of the ecology and morphology of our Sellaphora specimens will be necessary to clarify their correct taxonomic circumscription. Until then, we considered the specimen from the Rio Pardo basin S. pupula sensu lato. For comparison, the lectotype specimens of $S$. pupula designated by Mann (2001) are presented in figures 12-14.

Van Dam et al. (1994) stated that Nitzschia palea is a polysaprobic species indicator of hypereutraphentic states. In the Gravataí River (southern Brazil), this species was found in all samples taken from upper to lower reaches, although the highest densities were observed in the less-polluted courses (Salomoni et al., 2006). Lobo et al. (2004b) found that N. palea and $S$. pupula sensu lato were highly abundant in all samples from the Pardinho River, southern Brazil, during autumn 2002, when the water was heavily polluted. Additionally, Schneck et al. (2007) found N. palea and Luticola goeppertiana to be representative of southern Brazilian eutrophic waters in a high altitude stream $(\sim 1000$ m.a.s.l.), which was impacted by fish farming, with a significant downstream increase in nutrients and total solids levels. Analogous results were also found by Bruno et al. (2003) while studying planktonic diatom communities in a region of the Cuarto River, Argentina, which were under strong anthropogenic influence. Finally, Bellinger et al. (2006) reinforced the validity of
$N$. palea as an indicator of phosphorous enrichment in tropical streams from east Africa.

In lotic systems from southern Brazil, $N$. palea was classified as having an average tolerance for eutrophication (Lobo et al., 2004a) and a high tolerance for organic pollution (Lobo et al., 2002). However, Krammer \& Lange-Bertalot (1988) pointed out that $N$. palea has a wide range of tolerance for organic pollution, from mesosaprobic to polysaprobic conditions, with an ecological optimum in highly polluted waters. It is important to note that $N$. palea belongs to the Nitzschia section Lanceolatae Grunow, one of the most problematic sections within the genus due to the lack of distinct taxonomical criteria under light microscopy (Kobayasi, 1985; Tudesque et al., 2008). In fact, as Trobajo \& Cox (2006) stated, there is considerable overlap in the diagnostic morphological characteristics of $N$. palea and N. palea var. debilis (Kützing) Grunow, where the main difference between both taxa is valve width. This situation has lead to the misuse of these taxa in diatom-based environmental assessment studies. For example, Lange-Bertalot (1980) considers $N$. palea tolerant of organic pollution and N. palea var. debilis tolerant only of slightly polluted waters. According to Lobo et al. (2004b), N. palea was highly abundant in all samples collected in the Pardinho River, from upper to lower reaches, though the highest densities were observed in the lower courses. These results suggest that the specimens identified in southern Brazil probably represent species complexes rather than discrete taxa. Therefore, more detailed taxonomical, ecological and genetic investigations have to be done to clarify this problem.

Torrisi \& Dell'Uomo (2006), while working in rivers in central Italy, found Nitzschia clausii abundant in the lower reaches, which are classified as bad to very bad water-quality bodies for organic matter, nutrients and mineral salts. According to Krammer \& Lange-Bertalot (1988), this species is tolerant up to $\alpha$-mesosaprobic conditions (heavily polluted) and can live in inland waters with high electrolyte contents.

Nitzschia nana is a typical saprophilous species, according to Watanabe et al. (1990), and is also found in mesotrophic to eutrophic waters 


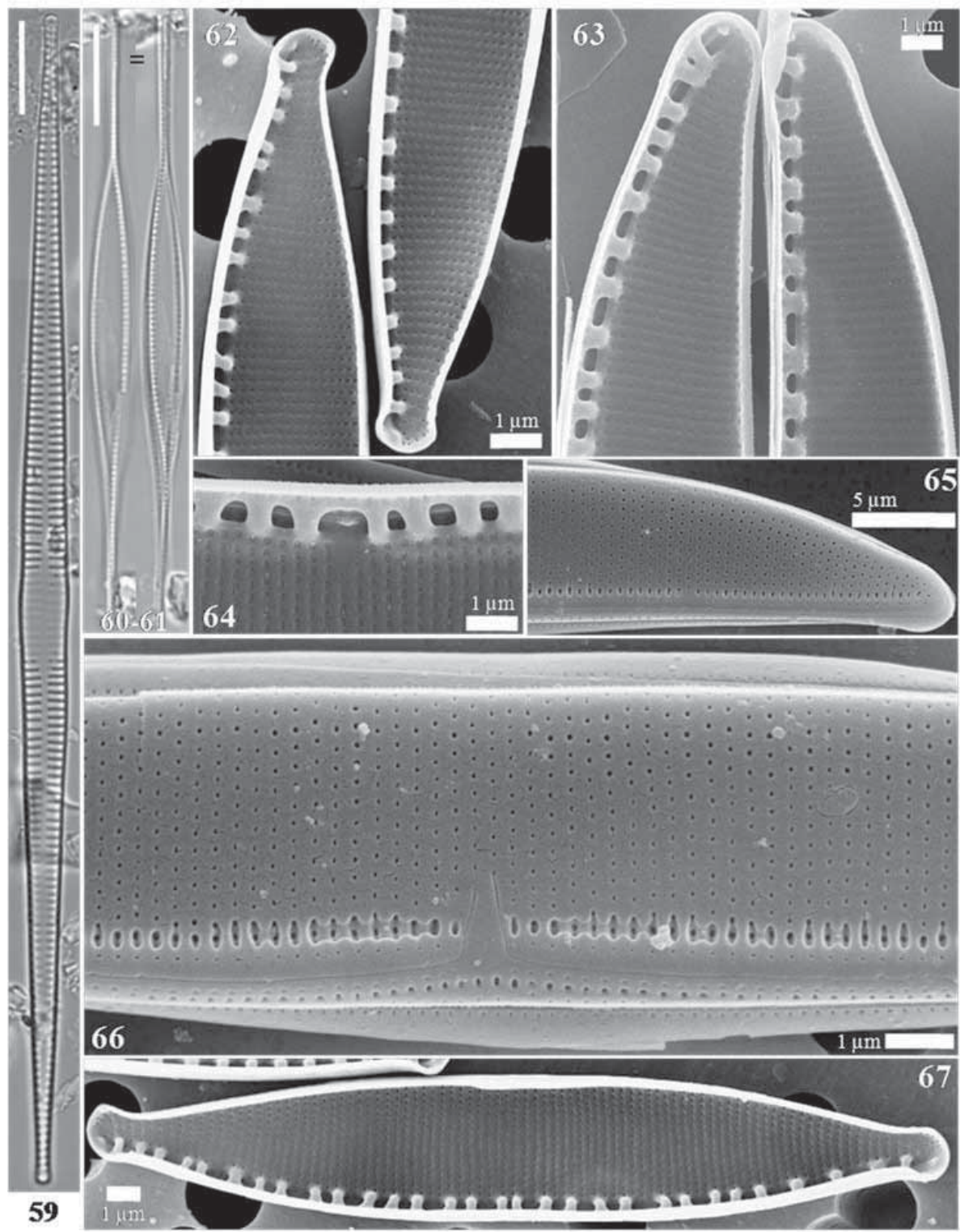

Figures 59-67. LM. Figs 62-67: SEM. 59: Ulnaria acus. 60-61: Nitzschia acicularis. 62: Internal valve apices view of $N$. palea, thin, slightly capitate valve ends, fairly irregular distance between fibulae, parallel uniseriate striae and small, rounded areolae openings. 63-66: $N$. clausii. 63: Internal apices view. 64: Internal view of the central area with the central nodule and more separated central fibulae. 65: External view. 66: External view of the central area, proximal raphe ends strongly bent in the same direction, very thin raphe slit, parallel uniseriate striae and small areolae. 67: Internal view of $N$. palea, linear-lanceolate shaped-valve, rostrate valve ends, parallel striae, irregular distance between fibulae and raphe filiform ending in a reduced helictoglossa. LM. Figs 62-67: SEM. 59: Ulnaria acus. 60-61: Nitzschia acicularis. 62: Vista apical interna de la valva de N. palea; con un extremo fino y suavemente capitado; la distancia entre las fíbulas bastante irregular; las estrías son uniseriadas y paralelas; la apertura de las areolas pequeña y redondeada. 63-66: N. clausii. 63: Vista apical interna. 64: Vista interna del área central, con nódulo central y fíbulas centrales más separadas. 65: Vista externa. 66: Vista externa del área central; extremidades proximales del rafe fuertemente curvadas hacia la misma dirección; apertura del rafe muy fina; estrías paralelas uniseriadas. Areolas pequeñas. 67: Vista interna de N. palea; valva con forma linear-lanceolada; extremos de la valva rostrados; estrías paralelas; distancia entre fíbulas irregular; rafe filiforme terminado en una helictoglosa reducida. 

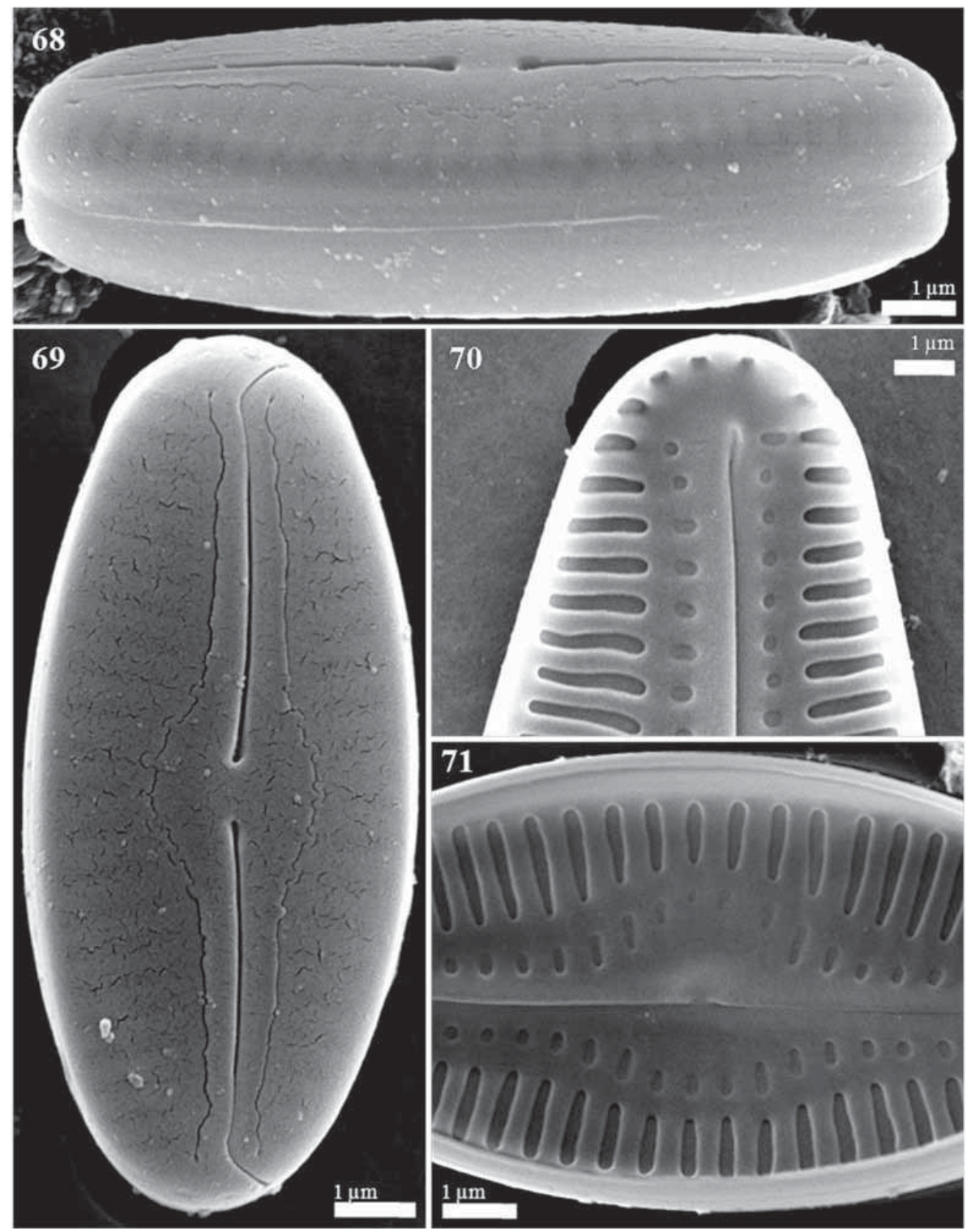

Figures 68-71. Images from scanning electron microscopy of Fallacia monoculata. 68: External oblique valve view with thin rectangular frustule in girdle view. 69: General valve shape elliptical with bluntly rounded apices; a thin sheet of silica (conopeum) covers the entire areolae portion of the valve. 70: Internal valve view of the pole, coarse, round to elongate areolae openings with one longitudinal row of very elongate areolae at the side of the edge of the valve plan and distal raphe endings that are weakly raised inside of the valve stopping straight in the helictoglossae. 71: Internal view of central area, one row of slightly elongate areolae at the side of the sternum, virgae slightly larger than striae, filiform raphe, weakly raised central nodule and central raphe endings shortly bent internally. Imágenes de microscopia electrónica de barrido de Fallacia monoculata. 68: Vista externa oblicua de la valva; frústulo en vista pleural rectangular, fino. 69: Forma general de la valva, elíptica con ápices redondeados. Una fina lámina de sílice (conopeo) cubre toda la porción de la valva con areolas. 70: Vista interna del polo de la valva; apertura gruesa de las areolas; redondeada a elongada, una fila longitudinal de areolas muy elongadas al lado del margen del plano valvar; el extremo distal del rafe aparece apenas elevado dentro de la valva, terminando recto en una helictoglosa. 71: Vista interna del área central; una fila de areolas suavemente elongadas del lado del esterno; virgae algo más largas que las estrías; rafe filiforme; nódulo central inconspicuo; extremo central del rafe poco curvado internamente. 

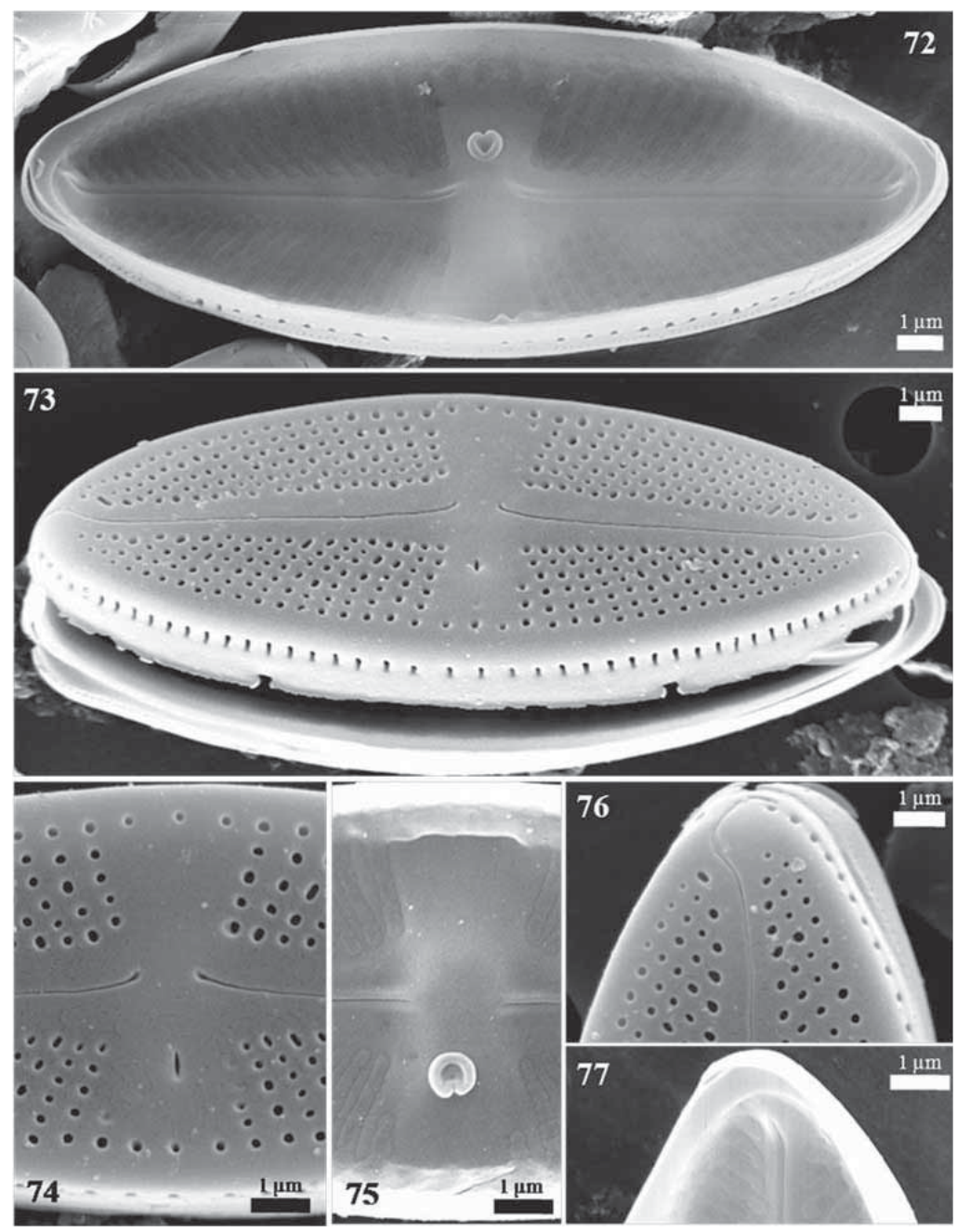

Figures 72-77. Images from scanning electron microscopy of Luticola goeppertiana. 72: Internal view of the valve, internal closing membranes, virgae thinner than striae, lanceolate axial area (sternum) and transapically butterfly-shaped central area. 73: External view of the valve, valve with broadly elliptic-lanceolate with cuneate rounded apices, markedly radiate striae composed of two to five areolae, striae reduced to one areola at the level of the central area, large and rounded areolae openings, striae interrupted at the level of the valve face/mantle transition, one line of areolae on the mantle, one large, transapically elongated stigma and coaxial, filiform, straight, central raphe. 74: Detail of external central area with drop-shaped central raphe endings that are curved to the side opposite the stigma. 75: Detail of internal central area, detail of round and prominently lipped stigma, central nodule with helictoglossae and sternum slightly raised and straight and slit-shaped central raphe endings. 76: Detail of external apical area with terminal raphe endings that are strongly hooked and extended to the mantle. 77: Detail of internal apical area with terminal raphe endings that are slightly curved to the same side as the proximal endings. Imágenes de microscopia electrónica de barrido de Luticola goeppertiana. 72: Vista interna de la valva; membranas internas cerradas; virgae más finas que las estrías; área axial (esterno) lanceolada; área central en forma de mariposa transapicalmente. 73: Vista externa de la valva; elíptica-lanceolada, con ápices cuneados redondeados; estrías marcadamente radiadas, compuesta de 2 a 5 areolas; estrías reducidas a una areola al nivel del área central; apertura de las areolas grande y redondeada; estrías interrumpidas al nivel de la transición de la parte valvar y el manto; línea de areolas sobre el manto; estigma grande, elongado transapicalmente; rafe coaxial, filiforme, recto, central. 74: Detalle del área externa central, extremidad central del rafe en forma de gota, curvada hacia el lado opuesto al estigma. 75: Detalle del área central interna; detalle del estigma redondeado y prominentemente labiado; nódulo central, helictoglosa y esterno ligeramente elevados; extremidades del rafe central rectas y en forma de ranura. 76: Detalle del área apical externa; extremidades del rafe terminal fuertemente curvadas extendidas hasta el manto. 77: Detalle del área interna apical; extremidades del rafe terminal suavemente curvadas hacia el mismo lado que la terminación proximal. 
(Lange-Bertalot \& Steindorf, 1996). Our study confirms the occurrence of $N$. nana in Brazilian waters with high electrolyte contents.

Fallacia monoculata was mainly distributed in the lower reaches of the Pardo and Pardinho rivers. Our specimens of $F$. monoculata from southern Brazil (Figs. 15-18, 20-23) are similar to Hustedt's type material of Navicula monoculata (Fig. 19. LM photograph of holotype material from a spring at the Babuna Pass, Macedonia) (Hustedt, 1945, pl. 41, Fig. 4). F. monoculata, which is associated with Nitzschia palea, Sellaphora pupula and N. clausii, was found to be abundant in highly polluted urban streams of the Pardinho basin. Ecologically, F. monoculata was not included in the Brazilian classification of trophic or organic pollution indicators (Lobo et al., 2002). Thus based on the results of this study and also the results of Souza \& Senna (2009) and Wetzel et al. (2006), F. monoculata may be considered highly tolerant of eutrophication and organic pollution, and we propose an indicative valor $(v i)$ of 5 and a saprobic value $(s)$ of 4 to be used in the calculation of the Biological Water Quality Index (BWQI), which was developed by Lobo et al. (2004a) for southern Brazilian rivers.

The present study thus provided evidence that epilithic diatom assemblages reflect land and water degradation by human activities in subtropical temperate streams, especially pollution by organic enrichment and eutrophication. The presence of nutrient tolerant taxa in impacted streams implies that anthropogenic activities are detrimental to these ecosystems and also corroborates the usefulness of benthic diatoms as ecological indicators for water-quality monitoring in southern Brazil.

\section{ACKNOWLEDGEMENTS}

The authors want to thank Dr. Zlatko Levkov (Friedrich Hustedt Diatom Collection, Bremerhaven, Germany), for providing Fallacia monoculata micrographs of Hustedt Type material. We would like to thank Christophe Bouillon (Public Research Center-Gabriel Lippmann, Belvaux, Luxembourg) for scanning electron microscopy technical assistance. Dr. Angelica Oliveira (Uni- versidade Federal de Santa Maria-UFSM, Brazil) kindly revised the English.

\section{REFERENCES}

AMERICAN PUBLIC HEALTH ASSOCIATION. 2005. Standard Methods for the Examination of Water and Wastewaters. 21 a ed. Washington: APHA. 1055 pp.

ARMITAGE, P. D. 1995. Behavior and ecology of adults. In: The performance of a new biological water quality score based on macroinvertebrates over a wide range of unpolluted running-water sites. P. D. Armitage, P. S. Cranston \& L. C. Pinder (eds.). Water Research, 17: 333-347.

BELLINGER, B. J., C. COCQUYT \& C. M. REILLY. 2006. Benthic diatoms as indicators of eutrophication in tropical streams. Hydrobiologia, 573: 7587.

BESZTERI, B., U. JOHN \& L. MEDLIN. 2007. An assessment of cryptic genetic diversity within the Cyclotella meneghiniana species complex (Bacillariophyta) based on nuclear and plastid genes, and amplified fragment length polymorphisms. Eur. J. Phycol., 42: 47-60.

BRUNO, E., A. MARTÍNEZ DE FABRICIUS \& M. E. LUQUE. 2003. Fitoplancton en un tramo del Río Cuarto con influencia antrópica. Bol. Soc. Argent. Bot., 38: 1-13.

CLEVE-EULER, A. 1953. Die Diatomeen von Schweden und Finnland. III. Kongl. Svenska Vetensk. Acad. Handl., 4: 1-255.

COCQUYT, C. 2000. Biogeography and species diversity of diatoms in the Northern Basin of Lake Tanganyika. Advances Ecol. Res., 31: 125-150.

CONAMA. 2005. Resolução CONAMA $n^{\circ} .357$ de 2005. Publicada no diário Oficial da União em 17 de março de 2005.

DENYS, L. 1991. A check-list of the diatoms in the Holocene deposits of the western Belgian coastal plain with a survey of their apparent ecological requirements. I. Introduction, ecological code and complete list. Prof. Pap. Geol. Surv. Belgium, 246: $1-41$.

ECTOR, L. \& F. RIMET. 2005. Using bioindicators to assess rivers in Europe: An overview. In: Modelling Community Structure in Freshwater Ecosystems. S. Lek, M. Scardi, P. F. M. Verdonschot, J. P. Descy \& Y. S. Park (eds.): 7-19. Springer Verlag, Berlin, Heidelberg. 
GOMEZ, N. \& M. LICURSI. 2001. The Pampean Diatom Index (IDP) for assessment of rivers and streams in Argentina. Aquatic Ecol., 35: 173-181.

HÅKANSSON, H. 2002. A compilation and evaluation of species in the genera Stephanodiscus, $\mathrm{Cy}$ clostephanos and Cyclotella with a new genus in the family Stephanodiscaceae. Diatom Res., 17: 1139.

HAMM, A. 1969. Die Ermittlung der Gewässergiiteklassen bei Fließgewässern nach dem Gewässergütesystem un Gewässergütenomogramm. Münchner Beitrdge zur AbwasserFischerei- and Flussbiologie, 15: 46-48.

HERMANY, G., A. SCHWARZBOLD, E. A. LOBO \& M. A. OLIVEIRA. 2006. Ecology of the epilithic diatom community in a low-order stream system of the Guaíba hydrographical region: subsidies to the environmental monitoring of southern Brazilian aquatic systems. Acta Limnol. Brasil., 18: 25-40.

HILL, M. O. \& H. G. GAUCH. 1980. Detrended correspondence analysis, an improved ordination technique. Vegetatio, 42: 47-58.

HUSTEDT, F. 1930-1966. Die Kieselalgen Deutschlands, Österreichs und der Schweiz unter Berücksichtigung der übrigen Länder Europas sowie der angrenzenden Meeresgebiete. In: KryptogamenFlora von Deutschland, Österreich und der Schweiz, Band 7. L. Rabenhorst (ed.): Teil 1: 1920, Teil 2: 1-845, Teil 3: 1-816. Akademische Verlagsgesellschaft m.b.H., Leipzig,

HUSTEDT, F. 1945. Diatomeen aus Seen und Quellgebieten der Balkan-Halbinsel. Arch. Hydrobiol., 40: 867-973.

KELLY, M. G. \& B. A. WHITTON. 1995. The trophic diatom index: a new index for monitoring eutrophication in rivers. J. Appl. Phycol., 7: 433-444.

KOBAYASI, H. 1985. Ultrastructural differences in certain taxonomically difficult species of Nitzschia section Lanceolatae in Japan. In: Origin and evolution of diversity in plants and plant community. H. Hara (ed.): 304-315. Academia, Tokyo.

KOBAYASI, H. \& S. MAYAMA. 1982. Most pollution tolerant diatoms of severely polluted rivers in the vicinity of Tokyo. Jap. J. Phycol., 30: 188-196.

KRAMMER, K. 2000. The genus Pinnularia. In: Diatoms of Europe. Diatoms of the European Inland Waters and Comparable Habitats 1. H. LangeBertalot (ed.): 1-703. Koeltz Scientific Books, Gantner Verlag, Koenigstein.
KRAMMER, K. \& H. LANGE-BERTALOT. 1986. Bacillariophyceae. 1. Teil: Naviculaceae. In: Süßwasserflora von Mitteleuropa, Bd. 2/1. H. Ettl, J. Gerloff, H. Heynig \& D. Mollenhauer (eds.): 1876. Gustav Fischer Verlag, Stuttgart.

KRAMMER, K. \& H. LANGE-BERTALOT. 1988. Bacillariophyceae. 2. Teil: Bacillariaceae, Epithemiaceae, Surirellaceae. In: Süßwasserflora von Mitteleuropa, Bd. 2/2. H. Ettl, J. Gerloff, H. Heynig \& D. Mollenhauer (eds.): 1-596. Gustav Fischer Verlag, Stuttgart.

KRAMMER, K. \& H. LANGE-BERTALOT. 1991a. Bacillariophyceae. 3. Teil: Centrales, Fragilariaceae, Eunotiaceae. In: Süßwasserflora von Mitteleuropa, Bd. 2/3. H. Ettl, J. Gerloff, H. Heynig \& D. Mollenhauer (eds.). Gustav Fischer Verlag, Stuttgart.

KRAMMER, K. \& H. LANGE-BERTALOT. 1991b. Bacillariophyceae. 4. Teil: Achnanthaceae, Kritische Ergänzungen zu Navicula (Lineolatae) und Gomphonema. In: Süßwasserflora von Mitteleuropa, Bd. 2/4. H. Ettl, G. Gärtner, J. Gerloff, H. Heynig \& D. Mollenhauer (eds.): 1-437. Gustav Fischer Verlag, Stuttgart.

LANGE-BERTALOT, H. 1980. New species, combinations and synonyms in the genus Nitzschia. Bacillaria, 3: 41-77.

LANGE-BERTALOT, H. \& A. STEINDORF. 1996. Rote Liste der limnischen Kieselalgen (Bacillariophyceae) Deutschlands. Schriftenreihe Vegetationsk., 28: 633-677.

LOBO, E. A. \& G. LEIGHTON. 1986. Estructuras de las fitocenosis planctónicas de los sistemas de desembocaduras de ríos y esteros de la Zona central de Chile. Revista Biol. Mar., 22: 1-29.

LOBO, E. A. \& V. L. CALLEGARO. 2000. Avaliação da qualidade de águas doces continentais com base em algas diatomáceas epilíticas: Enfoque metodológico. In: Avaliação e Controle da Drenagem Urbana. C. E. M. Tucci \& D. M. Marques (org.): 277-300. Porto Alegre: Ed. Universidade/UFRGS. 558 pp.

LOBO, E. A., V. L. M. CALlEGARO, M. A. OLIVEIRA, S. E. SALOMONI, S. SCHULER \& K. ASAI. 1996. Pollution tolerant diatoms from lotic systems in the Jacui Basin, Rio Grande do Sul, Brasil. Iheringia Ser. Bot., 47: 45-72.

LOBO, E. A., V. L. CALLEGARO \& P. BENDER. 2002. Utilização de algas diatomáceas epilíticas como indicadoras da qualidade da água em rios 
e arroios da Região Hidrográfica do Guaíba, RS, Brasil. Santa Cruz do Sul: EDUNISC. 127 pp.

LOBO, E. A., C. E. WETZEL \& D. BES. 2003. Avaliação da qualidade da água dos arroios Sampaio, Bonito e Grande, Municípío de Mato Leitão, RS, Brasil. Revista Tecnol. Brasil, 7: 39-53.

LOBO, E. A., V. L. CALLEGARO, G. HERMANY, D. BES, C. E. WETZEL \& M. A. OLIVEIRA. 2004a. Use of epilithic diatoms as bioindicator from lotic systems in Southern Brazil, with special emphasis on eutrophication. Acta Limnol. Brasil., 16: $25-40$.

LOBO, E. A., D. BES, L. TUDESQUE \& L. ECTOR. 2004b. Water quality assessment of the Pardinho River, RS, Brazil, using epilithic diatom assemblages and faecal coliforms as biological indicators. Vie \& Milieu, 53: 46-53.

LOBO, E. A., V. L. CALLEGARO, C. E. WETZEL, G. HERMANY \& D. BES. 2004c. Water quality study of Condor and Capivara streams, Porto Alegre municipal district, RS, Brazil, using epilithic diatoms biocenoses as bioindicators. Oceanol. Hydrobiol. Stud., 33: 77-93.

MALUF, J. R. T. 2000. A new classification for the State of Rio Grande do Sul. Revista Brasil. Agrometeor., 8: 141-150.

MANN, D. G. 1989. The species concept in diatoms: evidence for morphologically distinct, sympatric gamodemes in four epipelic species. Pl. Syst. Evol., 164: 215-237.

MANN, D. G. 2001. The systematics of the Sellaphora pupula complex: typification of $S$. pupula. In: Lange-Bertalot-Festschrift: Studies in Diatoms. R. Jahn, J. P. Kociolek, A. Witkowski \& P. Compère (eds.): 225-241. Gantner Verlag, Ruggell.

MANN, D. G., S. J. THOMAS \& K. M. EVANS. 2008. Revision of the diatom genus Sellaphora: a first account of the larger species in the British Isles. Fottea, 8: 15-78.

MCCUNE, B. \& M. J. MEFFORD. 1999. PC-ORD. Multivariate Analysis of Ecological Data, Version 4. Gleneden Beach: MjM Software Design. $237 \mathrm{pp}$.

METZELTIN, D. \& H. LANGE-BERTALOT. 1998. Tropische Diatomeen in Südamerika I. Iconogr. Diatomol., 5: 1-695.

METZELTIN, D. \& H. LANGE-BERTALOT. 2002. Diatoms from the "Island Continent" Madagascar. Iconogr. Diatomol., 11: 1-286.
METZELTIN, D. \& H. LANGE-BERTALOT. 2007. Tropical diatoms of South America II. Special remarks on biogeographic disjunction. Iconogr. Diatomol., 18: 1-876.

METZELTIN, D., H. LANGE-BERTALOT \& F. GARCÍA-RODRÍGUEZ. 2005. Diatoms of Uruguay. Iconogr. Diatomol., 15: 1-736.

OLIVEIRA, M. A., L. TORGAN, E. A. LOBO \& A. SCHWARZBOLD. 2001. Association of periphytic diatom species on artificial substrate in lotic environments in the Arroio Sampaio basin, RS, Brazil: relationships with abiotic variables. Revista Braz. J. Biol., 61: 523-540.

PATRICK, R. \& N. A. ROBERTS. 1979. Diatom communities in the Middle Atlantic States, U.S.A. Nova Hedwigia Beih., 64: 265-283.

ROCHA, A. 1992. Algae as biological indicators of water pollution. In: Algae and Environment: A General Approach. M. Cordeiro-Marino, M. T. P. Azevedo, C. L. Sant'Anna, N. Y. Tomita \& E. M. Plastino (eds.). p. 34-52. Sociedade Brasileira de Ficologia, CETEBS, São Paulo.

ROUND, F. E. 1972. Patterns of seasonal succession of freshwater epipelic algae. Brit. Phycol. J., 7: 213-220.

RUMRICH, U., H.LANGE-BERTALOT \& M. RUMRICH. 2000. Diatomeen der Anden. Von Venezuela bis Patagonien (Feurland). Iconogr. Diatomol., 9: 1-649.

SALOMONI, S. E., O. ROCHA, V. L. CALLEGARO \& E. A. LOBO. 2006. Epilithic diatoms as indicators of water quality in the Gravataí River, Rio Grande do Sul, Brazil. Hydrobiologia, 555: 233246.

SCHNECK, F., L. TORGAN \& A. SCHWARZBOLD. 2007. Epilithic diatom community in a high altitude stream impacted by fish farming in southern Brazil. Acta Limnol. Brasil., 19: 341-355.

SCHOEMAN, F. R. \& R. E. M. ARCHIBALD. 19761980. The Diatom Flora of Southern Africa. Pretoria: CSIRO. 579 pp.

SOUZA, M. G. \& P. A. SENNA. 2009. Diatomáceas epilíticas da subordem Sellaphorineae do rio do Monjolinho, São Carlos, SP, Brasil. Acta Botanica Brasílica, Brasil, 23(3): 618-629.

TORRISI, M. \& A. DELL'UOMO. 2006. Biological monitoring of some Apennine rivers (Central Italy) using the Diatom-based Eutrophication/Pollution Index (EPI-D) compared to other European diatom indices. Diatom Res., 21: 159-174. 
TROBAJO, R. \& E. J. COX. 2006. Examination of the type material of Nitzschia frustulum, N. palea and N. palea var. debilis. In: Proceedings of the 18th International Diatom Symposium. A. Witkowski (ed.): 431-445. Biopress Limited, Bristol.

TUDESQUE, L., F. RIMET \& L. ECTOR. 2008. A new taxon of the section Nitzschiae Lanceolatae Grunow: Nitzschia costei sp. nov. compared to $N$. fonticola Grunow, N. macedonica Hustedt, N. tropica Hustedt and related species. Diatom Res., 23: 483-501.

VAN DAM, H., A. MERTENS \& J. SINKELDAM. 1994. A coded checklist and ecological indicator values of freshwater diatoms from the Netherlands. Netherlands J. Aquatic Ecol., 28: 117-133.

WATANABE, T., K. ASAI, A. HOUKI \& T. YAMADA. 1990. Numerical spectrum by dominant diatom taxa in flowing and standing waters. In: Proceedings of the Tenth International Diatom
Symposium. H. Simola (ed.): 563-572. Koeltz Scientific Books, Koenigstein.

WETZEL, C. E., L. ECTOR \& E. A. LOBO. 2006. Diatomáceas epilíticas da Bacia Hidrográfica do Rio Pardo, RS, Brasil: Base taxonômica para a avaliação da qualidade da água em ambientes lóticos da região Sul do Brasil. In: Anais da $58^{a}$ Reunião Anual da SBPC, Florianópolis, SC, Brasil, julho/2006.

WETZEL, C. E., E. A. LOBO, M. A. OLIVEIRA, D. BES \& G. HERMANY. 2002. Diatomáceas epilíticas relacionadas a fatores ambientais em diferentes trechos dos rios Pardo e Pardinho, Bacia Hidrográfica do Rio Pardo, RS, Brasil: Resultados preliminares. Cad. Pesq. Sér. Biol. Brasil, 14: 17-38.

WOJTAL, A. Z. \& J. KWANDRANS. 2006. Diatoms of the Wyżyna Krakowsko-Czȩstochowska upland (Poland)-Coscinodiscophyceae (Thalassiosirophycidae). Polish Bot. J., 51: 177-207. 